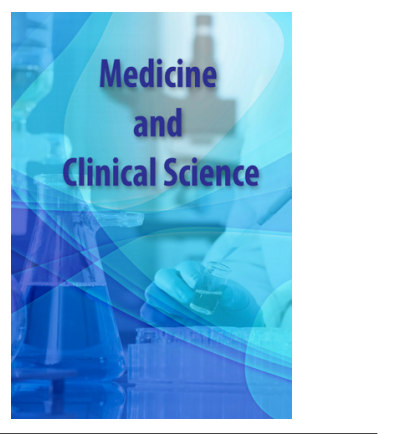

Correspondence

Jahanara Ainuddin

Professor of Obstetrics \& Gynecology Dow International Medical College, Dow University of Health Sciences, Karachi, Pakistan

Tel: +92-3012530961

E-mail: jahanaraainuddin@yahoo.com

- Received Date: 16 Nov 2019.

- Accepted Date: 30 Dec 2019.

- Publication Date: 03 Jan 2020

Keywords

Metformin, Pioglitazone, PCOS

\title{
Metformin versus pioglitazone in treatment of polycystic ovarian syndrome - A randomized control trial
}

\author{
Jahanara Ainuddin', Musfera Islam², Syeda Rabia ${ }^{3}$, Waqas Ahmed Farooqui ${ }^{4}$ \\ and Ashi Islam ${ }^{5}$ \\ 'Professor of Obstetrics \& Gynecology, Dow University of Health Sciences, Karachi, Pakistan \\ ${ }_{2}^{2}$ Postgraduate Resident, Department of Obstetrics and Gynecology, Dow University of Health Sciences, Karachi, Pakistan \\ ${ }^{3}$ Associate Professor, Department of Obstetrics and Gynecology, Dow University of Health Sciences, Karachi, Pakistan \\ ${ }^{4}$ Assistant Director \& Lecturer, Department of Research, Dow University of Health Sciences, Karachi, Pakistan \\ ${ }^{5}$ Consultant Gynecologist, Mamji Orthopedic \& General Hospital, Karachi, Pakistan
}

\begin{abstract}
Objective: To compare the efficacy of metformin versus pioglitazone in the treatment of polycystic ovarian syndrome. Methods: A prospective randomized controlled trial with parallel assignment was conducted in the outpatient Gynecology clinic of Dow University Hospital and Mamji Hospital, Karachi, Pakistan. One hundred and twenty patients with polycystic ovarian syndrome were selected and randomized into two groups with 60 patients receiving metformin in dose of 500mg TDS and 60 receiving pioglitazone $30 \mathrm{mg}$ daily. Clinical and biochemical parameters were evaluated at recruitment and at six months after treatment. Data was entered and analyzed through SPSS version 21. Results: Patients in both groups were Age and BMI matched. Significant reductions in fasting insulin levels, total testosterone and free androgen levels were found in both groups after treatment $(p<0.001)$. Clinical improvement in hirsutism and regularization of menstrual cycles was also observed in both groups $(p<0.001)$. However statistically significant reduction in BMI was observed in metformin group $(p<0.001)$ than in the pioglitazone group $(p=0.878)$. Conclusion: We conclude that both metformin and pioglitazone are effective in treatment of polycystic ovarian syndrome in terms of reduction of clinical and biochemical hyperandrogenism, regularization of menstrual cycles and restoration of ovulation. However metformin was found to be more effective in weight reduction
\end{abstract}

\section{Introduction}

(c) 2020 Science Excel. This is an openaccess article distributed under the terms of the Creative Commons Attribution 4.0 International license.
Polycystic Ovarian syndrome (PCOS) is the most commonest endocrine disorder of young girls women of reproductive age group affecting $8-13 \%$ of women in this age group [1,2]. PCOS is significant public health issue associated with reproductive (menstrual irregularly, hirsutism, sub fertility and miscarriages), metabolic and psychological problems $[1,3]$.

Polycystic ovarian syndrome is characterized by chronic anovulation, ovarian dysfunction, hyperandogenism and polycystic ovaries [4]. Polycystic ovarian syndrome is the most common cause of subfertility in the females [5]. Long term risks include type-2 diabetes mellitus, cardiovascular disorders and endometrial cancers [6]. Insulin resistance and hyperinsulinemia play an important role in the pathogenesis of PCOS [7]. Insulin sensitizers like pioglitazone and metformin will be useful in addition to life style modification for the treatment of PCOS [8]. These drugs have beneficial affects by decreasing insulin resistance and hyperandogenism thereby improving ovulation and pregnancy rates [9]. Metformin effects by increasing the level of [2] sex hormone binding globulin (SHBG) and thus a reduction in available free androgens [7]. Pioglitazone is a thiazolidinedione's (TZDS) which is a highly selective peroxisome proliferation activated receptor gamma (PPAR-r) and has been used for the treatment of diabetes. In PCOS there seems to be an alteration of PPAP-r gene, which plays an important role in fertility and metabolism through the effects of its various hypotypes $[10,11]$. Insulin resistance plays a key role is the pathogenesis of PCOS and is found not only in obese but in lean women with PCOS. PPAR-r agonist will help in reduction of androgen synthesis is ovaries in addition to reduction in peripheral insulin resistance $[12,13]$. There are no large scale published clinical trails to support the safety and efficacy of TZDs is the treatment of PCOS. TZDs are still not recommended by all guidelines as recognized treatment for PCOS. We conducted a randomized clinical trial in Pakistani (South Asian) population having a very high incidence of PCOS to compare the efficacy of metformin and pioglitazone in restoring ovulation and reduction of hirsutism and body weight and regularization of menstrual cycle.

\section{Methods}

This randomized clinical trial was conducted in the Obstetrics and Gynaecology Clinic of Dow University Hospital (DUHS) and Mamji Hospital Karachi from 1st September, 2018 till 28th February, 2019, The study was approved by the institutional review board and ethics committee of Dow University of Health Sciences Ref \# IRG-836/DUHS/Approval/2017/27. 
The trial is registered at clinicaltrials.gov will no NCT: 0375793. A total of 132 patients were enrolled in this study with PCOS between 18-40 years of age and randomly divided into two treatment groups using computer generated randomization number table. Informed written consent was obtained from all patients. PCOS was diagnosed according to revised Rotterdam's criteria as per (ESHRE) guidelines in 2018 [1]. Exclusion criteria include women with diabetes mellitus, known cardiac, liver or renal disease and hypertension, Girls less than 18 years of age, smokers and women taking other treatment for subfertility were also excluded. Pregnant and lactating women, women on oral contraceptive pills, women with adrenal and thyroid disorders and hyperprotactinemia were also excluded. One hundred and twenty patients were randomized in two groups Group A (Metformin) and Group B (Pioglitazone) with 60 patients in each group. At recruitment visit height, weight, BMI and blood pressure was recorded. History regarding regularity of menstrual cycle, oligomenorrhea was labeled as less than 8 cycles per year. Clinical examination was done and ferryman gallwey score calculated for each patient by given modified ferryman gallwey chart. FG score of more than 8 was taken as clinical hyperandogenism [1]. Blood samples were collected in a fasting state for fasting blood sugar, fasting insulin, serum testosterone, SHBG, serum prolactin, serum TSH, serum DHEAS and serum progesterone levels. Cut off level of serum fasting insulin was taken as $9.25 \mathrm{U} / \mathrm{ml}$ as hyperinsulinemia [14]. Free androgen index is calculated by using total testosterone and SHBG, and level of more than 4 is taken as increased free androgen level (biochemical hyperandogenism)serum progesterone level of less than $4 \mathrm{mg} / \mathrm{ml}$ is taken as anovulation, DHEAS level of (35-240 ug/dl) was taken as normal to include adrenal source of androgen. Ultrasound pelvis was done to diagnose polycystic ovaries, criteria used was more than 20 follicles of $2-9 \mathrm{~mm}$ in size in each ovary with ovarian volume of $\geq 10 \mathrm{ml}$. All these parameters were recorded at recruitment visit and repeated after 6 months and recorded. In Group A 66 patients were treated with metformin $500 \mathrm{mg}$ three times daily and in Group B pioglitazone was given as $30 \mathrm{mg}$ daily for 6 months. Vitamin supplements were given to patients in both groups. Healthy diet and daily exercise was advised to patients in both groups.

Outcome measures were recorded as hirsutism ferryman gallwey score, regularity of menstrual cycle, ovulation and reduction in body mass index.

\section{Statistical analysis}

Sample size was calculated using World Health Organization sample size calculated for two proportions. P1 $=42.9 \mathrm{P} 2=17.3$, level of significance at $5 \%$ and power of study at $80 \%$. Sample size of 120 patients with
60 in each group was calculated [6] estimated $10 \%$ dropout rate was considered and a total of 132 patients were selected for the study.

The data was analyzed on IBM SPSS version 21.0 and the results were presented before and after the treatment by drugs. For Quantitative variables like Age, BMI, FGS, FBS, SFI and Total testosterone, Mean \pm $\mathrm{SD}$ were reported. For Qualitative variables like regularity of menstrual cycle (RMC) and PCOs on Ultrasound, Frequency and percentage were reported. Statistical comparison for Quantitative variables was done using RM/Mixed Way ANOVA. For pair wise (between and within) comparison Post Hoc (Bonferroni) test was applied due to not have data normally distributed. For qualitative variables significance was reported using McNemar test. For qualitative outcomes at baseline if all patients i.e. $100 \%$ fall the p-value did not computed for those outcomes. However, the clinically difference in terms of \% has been reported. A p-value of 0.009 or less was considered statistically significant due to the Bonferroni test which reduced the significance level.

\section{Results}

A total of 120 patients with polycystic ovarian syndrome with 60 patients in metformin and 60 patients in pioglitazone group were included in this study. Mean age, BMI were similar in both pioglitazone and metformin group at study recruitment. A reduction in hirsutism in terms of improved Ferriman gallwey score were noted in both groups after treatment $(\mathrm{P} \leq 0.001)$ (Table 1$)$. Significant reductions in fasting blood sugar levels. Serum fasting insulin levels, total testosterone levels and free androgen index were found in both groups after treatment $\mathrm{P}$ $\leq 0.001$ (Table 1) however reduction in BMI was noted in metformin groups with mean difference of $2.5(\mathrm{O}:<0.001)$ which is significant. Reduction in $\mathrm{BMI}$ was not significant in pioglitazone group $(\mathrm{P}=0.878)$. Ovulation rate were also improved in both groups after treatment with serum progesterone levels of $27.6 \pm 19.0$ in metformin group and 33.6 \pm 21.0 in pioglitazone group both showing statistically significant improvement with $\mathrm{P} \leq 0.001$ (Table 1).

Within group comparison also revealed improvement in regularly of menstrual cycles, ovulation rates and ultrasound appearance of polycystic ovaries in both groups. $\mathrm{P}=0.01$ (Table 2). Comparison of clinical and biochemical characteristics between metformin and pioglitazone group revealed similar reductions in BMI, Ferriman gallwey score total testosterone levels, free androgen index and serum fasting insulin levels (Table 3). Patients in metformin group have high base line levels of total testosterone and free androgen index than in the Pioglitazone group but after treatment both drugs showed effective reduction in clinical and biochemical hyperandogenism with no significant deference $\mathrm{P} \geq 0.99$ (Table 3 ).

\begin{tabular}{|c|c|c|c|c|c|c|}
\hline \multirow[t]{2}{*}{ Characteristics } & \multicolumn{3}{|c|}{ Metformin $(\mathrm{N}=60)$} & \multicolumn{3}{|c|}{ Pioglitazone $(\mathrm{N}=60)$} \\
\hline & Baseline & After & $\begin{array}{c}\text { Mean Diff } \\
\text { (P-value) }\end{array}$ & Baseline & After & $\begin{array}{c}\text { Mean Diff } \\
\text { (P-value) }\end{array}$ \\
\hline \multicolumn{7}{|l|}{ Clinical } \\
\hline Age (Years) & $28.8 \pm 6.1$ & & $29.0 \pm 5.8$ & & & \\
\hline $\operatorname{BMI}\left(\mathrm{kg} / \mathrm{m}^{2}\right)$ & $27.5 \pm 4.2$ & $25.0 \pm 2.9$ & $2.5(<0.001 * *)$ & $26.3 \pm 3.8$ & $25.3 \pm 3.2$ & $0.95(0.878)$ \\
\hline FGS & $13.6 \pm 2.8$ & $7.1 \pm 1.7$ & $6.6\left(<0.001^{* *}\right)$ & $11.5 \pm 3.8$ & $7.7 \pm 5.3$ & $3.8\left(<0.001^{* *}\right)$ \\
\hline \multicolumn{7}{|l|}{ Biochemical } \\
\hline $\mathrm{fbs}$ & $97.7 \pm 7.2$ & $85.8 \pm 6.9$ & $11.9(<0.001 * *)$ & $94.8 \pm 7.6$ & $84.9 \pm 7.4$ & $9.9(<0.001 * *)$ \\
\hline sfi & $19.2 \pm 6.2$ & $9.1 \pm 2.6$ & $10.1(<0.001 * *)$ & $19.6 \pm 7.1$ & $8.9 \pm 2.6$ & $10.6\left(<0.001^{* *}\right)$ \\
\hline totalT & $6.1 \pm 1.9$ & $1.9 \pm 0.5$ & $4.3(<0.001 * *)$ & $4.5 \pm 2.0$ & $1.8 \pm 0.6$ & $2.7\left(<0.001^{* *}\right)$ \\
\hline fai & $9.2 \pm 1.5$ & $3.5 \pm 1.0$ & $5.7(<0.001 * *)$ & $7.5 \pm 2.1$ & $3.5 \pm 1.3$ & $3.98\left(<0.001^{* *}\right)$ \\
\hline progesterone & $3.2 \pm 0.9$ & $27.6 \pm 19.0$ & $-24.3(<0.001 * *)$ & $3.2 \pm 0.7$ & $33.6 \pm 21.0$ & $-30.4(<0.001 * *)$ \\
\hline DHEAS & $14.8 \pm 6.5$ & $12.6 \pm 5.5$ & $2.2(0.186)$ & $13.7 \pm 5.8$ & $11.3 \pm 4.5$ & $2.3(0.146)$ \\
\hline
\end{tabular}

**Significant at 0.009; BMI: Body mass index; FGS: Ferriman gallwey score; Fbs : Fasting blood sugar; Sfi: serum fasting insulin; Total T:

Total testosterone; Fai: free androgen index

Table1. Within group mean comparison of clinical \& biochemical characteristics 


\begin{tabular}{|c|c|c|c|c|c|c|}
\hline \multirow[t]{2}{*}{ Characteristics } & \multicolumn{3}{|c|}{$\operatorname{Metformin}(\mathrm{N}=60, \%)$} & \multicolumn{3}{|c|}{ Pioglitazone $(\mathrm{N}=60, \%)$} \\
\hline & Baseline & After & \% Diff (P-value) & Baseline & After & \% Diff (P-value) \\
\hline \multicolumn{7}{|l|}{ Clinical } \\
\hline RMC $(<8$ cycles $)$ & $54(90 \%)$ & $23(38 \%)$ & $52\left(<0.001^{* *}\right)$ & $58(97 \%)$ & $19(32 \%)$ & $65(<0.001 * *)$ \\
\hline $\mathrm{PCO}$ on $\mathrm{U} / \mathrm{S}$ & $57(95 \%)$ & $21(35 \%)$ & $60\left(<0.001^{* *}\right)$ & $59(98 \%)$ & $18(30 \%)$ & $68\left(<0.001^{* *}\right)$ \\
\hline
\end{tabular}

**Significant at 0.009; RMC: Regularity of menstrual cycle; PCO on U/S: polycystic ovaries on ultrasound

Table 2. Within Group Comparison of Clinical Characteristics

\begin{tabular}{|c|c|c|c|c|c|c|}
\hline \multirow[t]{2}{*}{ Characteristics } & \multicolumn{3}{|c|}{ Baseline } & \multicolumn{3}{|c|}{ After } \\
\hline & $\begin{array}{l}\text { Metformin } \\
(\mathrm{N}=60)\end{array}$ & $\begin{array}{c}\text { Pioglitazon } \\
(\mathrm{N}=60)\end{array}$ & $\begin{array}{c}\text { Mean Diff } \\
\text { (P-value) }\end{array}$ & $\begin{array}{l}\text { Metformin } \\
(\mathbf{N}=60)\end{array}$ & $\begin{array}{l}\text { Pioglitazone } \\
(\mathbf{N}=60)\end{array}$ & $\begin{array}{c}\text { Mean Diff } \\
\text { (P-value) }\end{array}$ \\
\hline \multicolumn{7}{|l|}{ Clinical } \\
\hline Age (Years) & $28.8 \pm 6.1$ & $29.0 \pm 5.8$ & $0.18(>0.99)$ & $28.8 \pm 6.1$ & $29.0 \pm 5.8$ & $0.18(>0.99)$ \\
\hline BMI $\left(\mathrm{kg} / \mathrm{m}^{2}\right)$ & $27.5 \pm 4.2$ & $26.3 \pm 3.8$ & $1.3(0.296)$ & $25.0 \pm 2.9$ & $25.3 \pm 3.2$ & $0.3(>0.99)$ \\
\hline FGS & $13.68 \pm 2.8$ & $11.5 \pm 3.8$ & $2.2(0.009 * *)$ & $7.1 \pm 1.7$ & $7.7 \pm 5.3$ & $0.63(>0.99)$ \\
\hline \multicolumn{7}{|l|}{ Biochemical } \\
\hline FBS & $97.7 \pm 7.2$ & $94.8 \pm 7.6$ & $2.9(0.180)$ & $85.8 \pm 6.9$ & $84.9 \pm 7.4$ & $0.95(>0.99)$ \\
\hline SFI & $19.2 \pm 6.2$ & $19.6 \pm 7.1$ & $0.33(>0.99)$ & $9.1 \pm 2.6$ & $8.9 \pm 2.6$ & $0.16(>0.99)$ \\
\hline Total T & $6.1 \pm 1.9$ & $4.5 \pm 2.0$ & $1.6(<0.001 * *)$ & $1.9 \pm 0.5$ & $1.8 \pm 0.6$ & $0.1(>0.99)$ \\
\hline FAI & $9.2 \pm 1.5$ & $7.5 \pm 2.1$ & $1.8(<0.001 * *)$ & $3.5 \pm 1.0$ & $3.5 \pm 1.3$ & $0.1(>0.99)$ \\
\hline Progesterone & $3.2 \pm 0.9$ & $3.2 \pm 0.7$ & $0.005(>0.99)$ & $27.6 \pm 19.0$ & $33.6 \pm 21.0$ & $6.05(0.122)$ \\
\hline DHEAS & $14.8 \pm 6.5$ & $13.7 \pm 5.8$ & $1.2(>0.99)$ & $12.6 \pm 5.5$ & $11.3 \pm 4.5$ & $1.25(>0.99)$ \\
\hline
\end{tabular}

**Significant at 0.009; BMI: Body mass index; FGS: Ferriman gallwey score; Fbs : Fasting blood sugar; SFI: serum fasting insulin; Total T:

Total testosterone; Fai: free androgen index

Table 3. Between group mean comparison of clinical \& biochemical characteristics

\section{Discussion}

This study was conducted to compare the efficacy and therapeutic effects of metformin and pioglitazone in women with PCOS. PCOS when fully expressed phenotypically is characterized by metabolic defects related to insulin resistance and hyperandogenism [15]. Insulin resistance associated fasting hyperinsulinemia and impaired response to oral glucose are present in about $30 \%$ of lean and $70 \%$ of obese PCOS women [16]. Our study was conducted to assess the effectiveness of metformin and pioglitazone in reduction of hyperinsulinemia and hyperandogenism and thus in regularization of menstrual cycles, improvement in clinical and biochemical hyperandogenism and restoration of ovulation in the back ground of local, ethnic, socioeconomic and environmental influences of our Pakistani population. Age and BMI matched patients with PCOS were recruited for the study in both groups, significant reduction in fasting blood sugar levels and fasting insulin level were found after treatment in both groups similar results were found in various other studies $[6,12,17]$. Reduction in BMI was observed more in women receiving metformin than in pioglitazone group in a study. An Indian study conducted in 2016 by Shahebrahimi K., showed observation with significally decreased waist circumference in metformin group with no significant change in pioglitazone group, similar observation was also noted by Romualdi et al., and other investigators. Ovulation rates were significally improved in our study in both groups, similar results of improvement in ovulatory function is demonstrated in other studies showing equal effectiveness of both drugs in restoring ovulation [20] However Razzaq M in her study reported pioglitazone to be more effective than metformin for ovulation induction in PCOS [21].

Both drugs were found to be equally effective in reduction of clinical and biochemical hyper androgen, restoration of ovulation and regularization of menstrual cycle in our study, However metformin was found to be more effective in weight reduction than Pioglitazone. It is recommended that both drugs can be used in PCOS out weighing the physician reference and patient tolerance and compliance with the drug. It is also recommended that pioglitazone should be stopped as soon as the patient achieves pregnancy as it is classified under category $\mathrm{C}$ drugs by FDA with possible teratogenic risks in developing fetus. However metformin being a category B drug can be continued in pregnancy because of the possible effects of decreasing first trimester pregnancy losses in PCOS women in pregnancy.

\section{Conclusions}

Both metformin and pioglitazone are effective in patients with PCOS in reducing hyperandogenism, restoration of ovulation in regularization of menstrual cycles. We cannot recommend one drug to be superior to other both being effective in treatment of PCOS and can be given as an alternative treatment in those patients who are unable to tolerate or non-complaint to one or other drug.

\section{Clinical Trail Identification No:}

\section{NCT 0375793}




\section{CONSORT 2010 Flow Diagram}

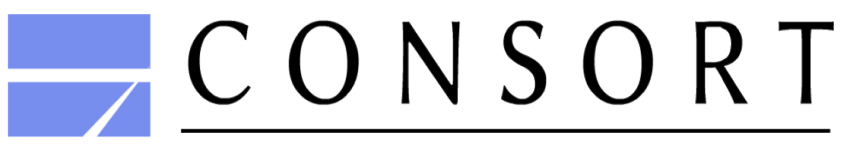

TRANSPARENT REPORTING of TRIALS

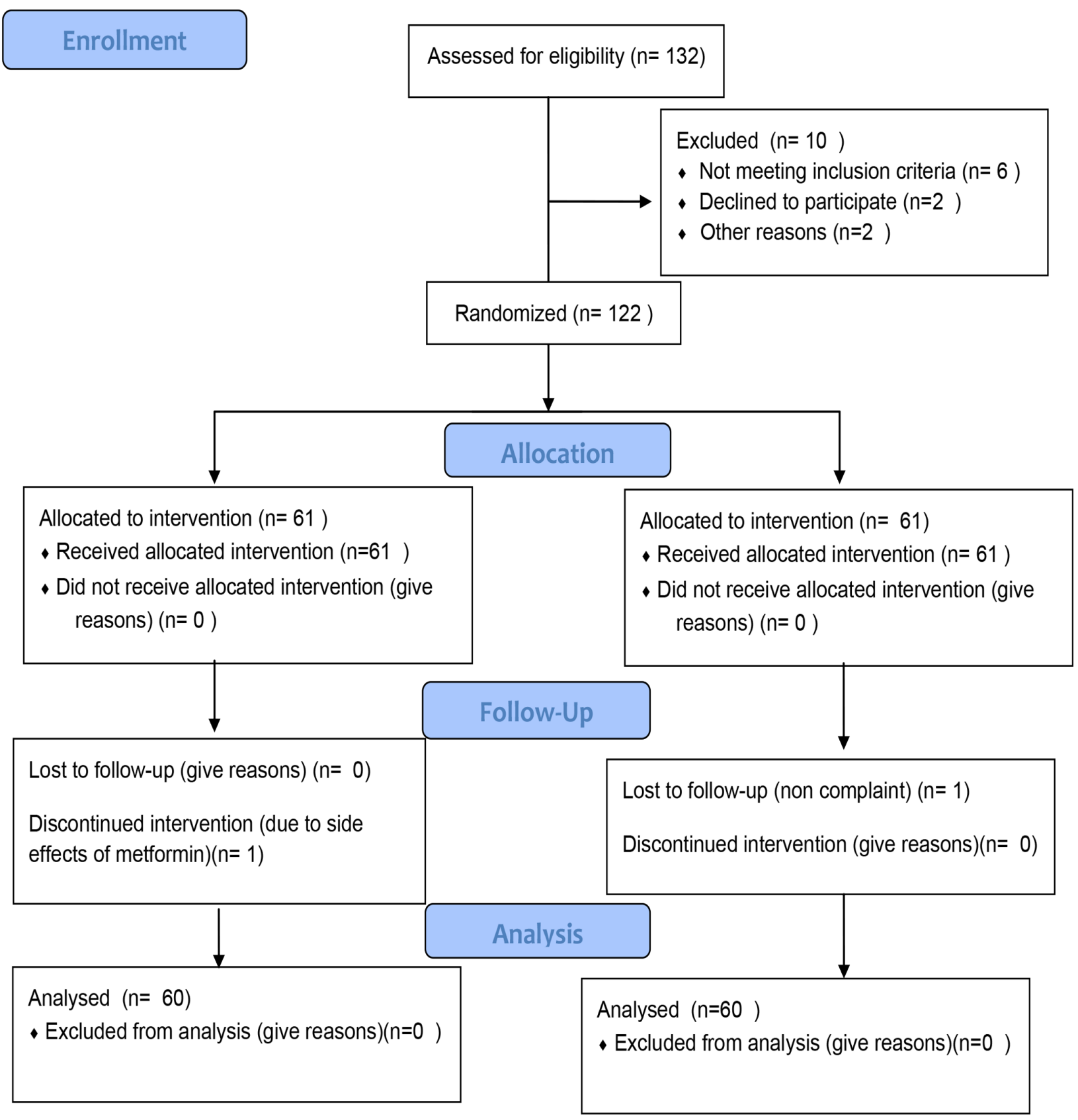

Figure 1. CONSORT 2010 Flow Diagram 


\section{References}

1. International evidence - based guideline for the assessment and management of polycystic ovary syndrome (Eshre).

2. Bdag G. The prevalence and pheutypic features of Polycystic ovary syndrome - A systemic review and Meta-analysis. Hum Repro. 2016; 31(12):.2841-2855.

3. Moran L, et al., Polycystic ovary syndrome: a biopsy Cho social understanding in young women to improve knowledge and treatment options. Journal of Psychosomatic obstetrics and Gynecology. 2010; 31(1):P.24-31

4. LauristenMP, Beutzen JG, Pinborg A, Loft A et al., The prevalence of Polycystic ovary syndrome in a normal population according to the Rotterdam criteria versus refised criteria including anti-Mullerian harmone. Hum Repro. 2014; 29(04): 791-801.

5. Baqai $Z$, Khanam M, Parveen $S$, et al., Prevalence of PCOS in infertile patients . Medical Channel, 2012; 16(03):437-440

6. Shaherbrahimi K, Jalillian N, Bazgir N, et al. Comparison in Polycystic ovary synderome. Indian Endocrinol Metab, 2016; 20(6): 805-809.

7. Brettenhalee N, Geytaer CD, Huber PR, et al., Effect of insulin reusitizer Pioglitazone on Insulin resistence, Hyperandrogenism and ovalatary dysfunction in women with polycystic ovary syndrome. J Clim Endocrinol Metab 2004; 89: 3835-40.

8. Naka KK, Kalantaridov SN, Kravariti M, et al; Effect of the insulin sensitizers metformin and Pioglitazone' on endothelial function in young women with polycystic ovary syndrome: A prospective randomized study fertility and sterility. 2011; 95(01): 203-209.

9. Ota H, Goto T, Toshioka $\mathrm{T}$, et al. Succesful pregnancies treated with Pioglitazone in infertile patients with polycystic ovary syndrome. Fertility and sterility. 2008; 90(3): 709-4013.

10. Foroment P, Gizard F, Staels B, Dupout J, Magnet P. A rote of PPAP-r in reproduction. Med Scci. 2005; 21(5):507-511.

11. Yu Y, Wu Y, Huang Q. Comparison of the effect between Pioglitazone and metformin in treating patients with PCOS: a meta-analysis. Arch gynecol Obstet. 2017; 294(4): 661-677.
12. Doganay $M$, Ozyer SS, Var T, et al. Association between adipocyte fatty acid binding protein and clinical parameters in polycystic ovary syndrome. Arch Gynecol Obstet. 2015:291(2): 447-450.

13. Schoppee PD, Garmey JC, Veldhuis JD. Putative activations of the peroxisome proliferate-activated receptor gamma impauis androgen and enhances progesterone biosynthesis in primary cutturs porcine theca cells. Biol Report. 2002; 66(1):190-198.

14. Hydri MZ, Basit A, Fawwad A, et al., Detecting insulin resistance in Pakistani subjects by fasting blood samples. The Open Diabetes journal. 2012; 5(1): 20-24.

15. Akram M, Roohi N. Endocrine correlates of polycystic ovary syndrome in Pakistani women. JCPSP. 2015; 25(1):22-26.

16. Legro RS, Kunsetman AP, Dodson WC, et al. Prevalenceand predictors of risk for type 2 diabetes and impaired glucose tolerance in polycystic ovary syndrome: A prospective, controlled study in 254 affected women. J CLin Endocrinol Metab. 1999; 84:165-169.

17. Sohervardi SM, Nosouhi F, Khalilzade SH, Kafaie P, Karims zarohi M, Halvali I etal). Evaluating the effect of insulin sensitizers metformin and pioglitazone alone and in combination on women with polycystic ovary syndromne : an RCT. Int J Reprod BioMed. 2016; 14(12): 743-754.

18. Romuvaldi D, Guido M, Ciampelli M, Giuliani M, Leoni F, Perri C, eatl. Slecetive effects of pioglitazoneon insulin and androgen abnormalitiesin narmo and hyperinsulemic obese patients with polycystic ovary syndrome. Hum Repro. 2003;18: 1210-8.

19. Du Q, Yang S, Wang Y, Wu B etal; effects of thiazolidinediones on plycystic ovary syndrome : : A mMeta-analysis of Randamized placebo - controlled trails. Adv ther. 2012; 29(91):763-774.

20. Shah S. Metformin and pioglitazone in polycystic ovarian syndrome: $\mathrm{A}$ comparative study. J obs \& Gynae of India. 2012; 62(5):551-556.

21. Razzaq M, Wabasa M, Aslam H. Comparison of efficacy of Metformin versus pioglitazone in patients with polycystic ovarian syndrome. 2016; 4(10):1421-1424. 